\title{
Bone Histomorphometry in Children with Newly Diagnosed Acute Lymphoblastic Leukemia
}

\author{
JAN A. LEEUW, JAN KOUDSTAAL, JANNEKE WIERSEMA-BUIST, WILLEM A. KAMPS, AND \\ WIM TIMENS
}

Department of Pediatrics [J.A.L., W.A.K.], Division of Pediatric Oncology/Hematology, Beatrix Children's Hospital, University Hospital Groningen, 9700 RB Groningen, The Netherlands; Department of Pathology [J.K., J.W.-B., W.T.], University Hospital Groningen, 9700 RB Groningen, The Netherlands

\begin{abstract}
The objective of this study was to obtain insight into bone formation and resorption in children with newly diagnosed untreated acute lymphoblastic leukemia (ALL). In 23 consecutive children with ALL, a bone biopsy was taken from the crista iliaca posterior under ketamine anesthesia, together with the diagnostic marrow aspiration, before any treatment was given. Histomorphometric assessment was done of bone volume, bone area, trabecular thickness, osteoid volume, osteoid area, osteoid width, number of osteoblasts, erosion area, and number of osteoclasts. Data were analyzed in age groups under and over $10 \mathrm{y}$ and compared with biopsies from 15 children, obtained during the work-up for other malignancies (only patients without bone marrow involvement were included). In ALL patients, bone volume and trabecular thickness were decreased in children $<10$ y. In patients $>10 \mathrm{y}$, these parameters were not significantly different from the controls; bone densitometry showed no significant loss of bone in patients $>10 \mathrm{y}$ as well. Numbers of
\end{abstract}

\section{ABSTRACT}

osteoblasts and osteoid surface occupied with osteoblasts were reduced in both age groups, as was the number of resorbing osteoclasts. No indications of osteomalacia were found. Childhood ALL results in a reduced number of both osteoblasts and osteoclasts, with a subsequent reduced osteoid formation and reduced bone resorption. This leads to a reduced bone volume and trabecular thickness, especially in younger children. In adolescents, the disturbance of bone (re)modeling is less serious, probably because of the strong stimulus on bone formation of sex hormones. The skeletal impairment at diagnosis is potentially reversible. (Pediatr Res 54: 814-818, 2003)
Abbreviations
ALL, acute lymphoblastic leukemia
BMD, bone mineral density
PTH, parathyroid hormone

Bone and joint pain or limping are common presenting symptoms of childhood acute lymphoblastic leukemia (ALL). Diffuse osteoporosis may be found, and even compression fractures do occur, mainly of the spine. The mechanisms that give rise to these phenomena have not been fully understood. Histomorphometric case reports have suggested increased resorption of bone or defective mineralization $(1,2)$. Circulating biochemical markers of bone metabolism are consistent with a low bone turnover (3).

To obtain more insight into the mechanisms, we studied the bone histomorphometry of 23 children with newly diagnosed ALL before any specific anticancer treatment was given. These histomorphometric data were compared with those of 15 control bone biopsies obtained for diagnostic reasons from chil-

Received November 12, 2001; accepted February 12, 2003.

Correspondence: Jan A. Leeuw, MD, Department of Pediatrics, Division of Pediatric Oncology/Hematology, University Hospital Groningen, Beatrix Children's Hospital, P.O. Box 30.001, 9700 RB Groningen, The Netherlands; email: j.a.leeuw@bkk.azg.nl

This study was supported by a grant from the Foundation for Pediatric Oncology Research Groningen.

DOI: 10.1203/01.PDR.0000090929.16450.DE dren with established or suspected malignancies without bone or bone marrow involvement.

\section{METHODS}

Patients. Twenty-nine children with ALL were assessed at diagnosis, before any treatment was given. These were consecutive cases in a time period of $2.5 \mathrm{y}$. Biopsies of six children were technically insufficient. Control biopsies were obtained for diagnostic reasons from children with established or suspected malignancies. Only patients without bone or bone marrow involvement were included. Patients and control subjects were divided into age groups: under and over $10 \mathrm{y}$ of age, for purpose of determining potential differences as a result of sex hormones in pubertal children (Tanner stage was not consequently recorded); 18 patients and nine control subjects were younger than $10 \mathrm{y}(1.8-9.9 \mathrm{y})$; the remaining (five patients and six control subjects) were 12.5-17 y of age. Some histomorphometric data about healthy children in the same age groups have been published (4-6). Patient and control characteristics are listed in Tables 1 and 2 . 
Table 1. Characteristics of ALL patients

\begin{tabular}{|c|c|c|c|c|c|}
\hline & \multirow{2}{*}{$\begin{array}{l}\text { Age (y; [median, } \\
\text { range] })\end{array}$} & \multirow{2}{*}{$\begin{array}{l}\text { Leucocytes } \times 10^{9} / \mathrm{L} \\
(\text { median, range })\end{array}$} & \multicolumn{3}{|c|}{ Leukemic cell immunophenotype } \\
\hline & & & Pre-pre-B & Pre-B & $\mathrm{T}$ \\
\hline Patients $<10$ y $(n=18)$ & $3.5(1.8-9.0)$ & $9.5(1.8-387.0)$ & 11 & 6 & 1 \\
\hline
\end{tabular}

Table 2. Characteristics of control subjects

\begin{tabular}{lcc}
\hline & $\begin{array}{c}\text { Control subjects } \\
<10 \mathrm{y}(n=9)\end{array}$ & $\begin{array}{c}\text { Control subjects } \\
>10 \mathrm{y}(n=6)\end{array}$ \\
\hline Age (y, median, range) & $4.8(2.3-9.9)$ & $13.4(12.1-15.8)$ \\
Diagnosis & 1 & \\
$\quad$ Retinoblastoma & 2 & \\
$\quad$ Rhabdomyosarcoma & 3 & 1 \\
$\quad$ Medulloblastoma & 2 & 1 \\
$\quad$ Non-H-lymphoma & & 2 \\
Hodgkin lymphoma & & 1 \\
$\quad$ Olfactory neuroblastoma & & \\
$\quad$ Undifferentiated renal cell & & 1 \\
$\quad$ carcinoma & 1 & \\
Cat scratch disease & & \\
\hline
\end{tabular}

This study was approved by the institutional ethical review board. Written informed consent was obtained in accordance with the institutional rules.

Histomorphometry. During ketamine narcosis, a bone biopsy was taken from the posterior iliac crest with a 2-mm core trephine, via a vertical approach. Biopsy samples were fixed in $2 \%$ paraformaldehyde and embedded in glycol methacrylate resin (7). Sections $(2 \mu \mathrm{m})$ were cut on a Reichert-Jung Supercut plastic microtome. Five sections with an interspace of 100 $\mu \mathrm{m}$ were used for histomorphometry and stained with solochrome cyanine, discriminating between mineralized bone (light blue staining) and osteoid (light red-orange staining) (8), combined with tartrate-resistant acid phosphatase, identifying osteoclasts by a dark red staining. Osteoblasts, actively making osteoid, were morphologically recognized by their cubical or cylindrical shape. Osteoid covered by flat lining cells was considered inactive. An example of the solochrome cyanine/ tartrate-resistant acid phosphatase staining is given in Figure 1. Five consecutive sections were stained for alkaline phosphatase to facilitate the recognition of osteoblasts.

Histomorphometry uses stereologic principles that state that in an infinite thin section, two-dimensional quantities such as area and perimeter can be extrapolated to three-dimensional quantities such as volume and surface area (9). For measuring the two-dimensional profiles, a microscopic image was projected onto a television monitor, superimposed by a grid containing 36 points and four sinusoid test lines of known length. Microscope objective and monitor produced a magnification of 2500. Fifty fields per section were counted. Counting more fields did not change means of outcome (data not shown). Extrapolation from the fraction of points overlying particular structures to a volume fraction and extrapolation from the counts of intersections between the test lines and particular structures to three-dimensional surface areas was done using formulas as described by Schenk (10). The following parameters of trabecular bone were assessed: bone volume (percentage of bone tissue between the subcortical envelopes),

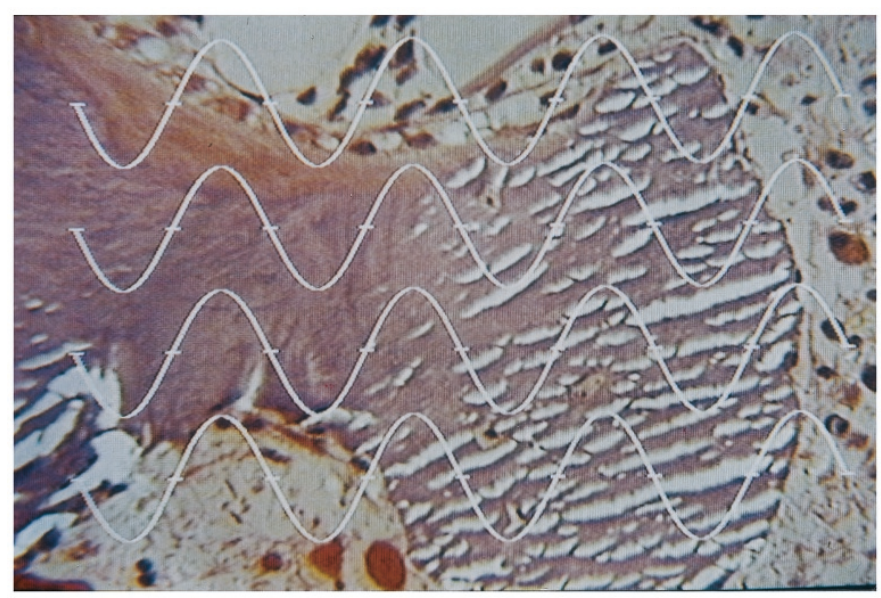

Figure 1. Example of a monitor image, showing the main features of bone turnover. The greater part of this section consists of trabecular bone (purple blue); only a small amount of the surrounding bone marrow is visible. The upper trabecular surface shows at the left a seam of brown-orange osteoid, recently produced by cubical osteoblasts (brown). At the lower left trabecular surface, a lacuna is visible, partly filled with a red stained osteoclast (active resorption). The bone surface at the right hand of the picture shows neither bone formation nor resorption, so-called neutral bone surface. Superimposed on the microscopy field is the histomorphometry grid with four sinusoid test lines and 36 test points.

bone surface (surface of trabecular bone tissue, osteoid included, related to the total volume between the cortices), trabecular thickness (mean thickness of the trabeculae), osteoid volume (volume of osteoid as percentage of the total trabecular bone volume), active osteoid surface (percentage of total trabecular surfaces covered with osteoid with a border of osteoblasts), inactive osteoid surface (percentage of trabecular osteoid seams covered by flat lining cells), osteoid thickness (mean thickness of osteoid seams), number of osteoblasts (counts per field of vision), active erosion surface (percentage of total trabecular surfaces where erosion by osteoclasts is actively continuing), inactive erosion surface (percentage of total trabecular surfaces where resorption has ceased and osteoclasts have disappeared), and number of osteoclasts (counts per field of vision). The nomenclature used in this paper is according to the report of the ASBMR Histomorphometry Nomenclature Committee (11).

Bone densitometry. Bone mineral density (BMD) of the lumbar vertebrae (L1-L4) was measured, using the dualenergy x-ray absorptiometry method (Hologic 1000) and expressed as $z$ scores for comparison across all ages and sexes.

Statistics. Data between groups were compared, using the Mann-Whitney test, a nonparametric test for comparing data from two independent groups. For further estimating the actual bone formation, Spearman correlation tests (calculating rank correlation coefficients) were done between the extent of the osteoblast-osteoid interface and the osteoid volume (10); $p<$ 
Table 3. Histomorphometry results of ALL patients and control subjects: parameters of bone volume and structure

\begin{tabular}{lccc}
\hline & $\begin{array}{c}\text { Trabecular bone volume } \\
{\left[\frac{\mathrm{mm}^{3}}{\mathrm{~mm}^{3}} \times 100\right]} \\
(\text { median, range) }\end{array}$ & $\begin{array}{c}\text { Trabecular bone area } \\
\left(\mathrm{mm}^{2} / \mathrm{mm}^{3} ; \text { median, range) }\right.\end{array}$ & $\begin{array}{c}\text { Mean trabecular thickness } \\
(\mu \mathrm{m} ; \text { median, range })\end{array}$ \\
\hline Patients $<10 \mathrm{y}(n=18)$ & $17.3(13.2-25.3)$ & $21.7(13.4-38.8)$ & $122.2(82.0-169.8)$ \\
Controls $<10 \mathrm{y}(n=9)$ & $20.7(16.6-23.1)$ & $17.5(14.4-28.4)$ & $146.1(100.2-166.6)$ \\
$p$ value & 0.04 & $\mathrm{NS}$ & 0.03 \\
Patients $>10 \mathrm{y}(n=5)$ & $19.8(15.6-28.5)$ & $16.3(12.2-27.2)$ & $141.3(118.2-168.7)$ \\
Controls $>10 \mathrm{y}(n=6)$ & $27.1(22.3-34.8)$ & $14.0(10.6-18.8)$ & $167.7(139.8-205.1)$ \\
$p$ value & $\mathrm{NS}(0.07)$ & $\mathrm{NS}$ & $\mathrm{NS}$ \\
\hline
\end{tabular}

0.05 was considered significant. For comparison BMD with reference values, a one-sample $t$ test was used, revealing whether the $z$ scores differed significantly from zero, the mean of the standard normal distribution of the reference values.

\section{RESULTS}

Parameters of bone volume and structure are shown in Table 3. In the ALL patients $<10 \mathrm{y}$, a significant decrease of the trabecular bone volume was found. The bone surface in relation to the total bone mass was not different between patients and control subjects, but the thickness of the bone trabeculae was diminished. ALL patients $>10 \mathrm{y}$ did not differ significantly on any of the parameters. In the control subjects $<10 \mathrm{y}$, there were some patients with a low trabecular bone volume, not exclusively associated with specific diagnoses.

Parameters of bone resorption are shown in Table 4. The percentage of total eroded bone (inactive and active) with respect to the total bone surface was not different, but the percentage of the active eroded surface was significantly decreased in ALL patients ( $<10 \mathrm{y}, p<0.01 ;>10 \mathrm{y}, p=0.04)$, as were the numbers of osteoclasts per field of vision $(<10 \mathrm{y}$, $p=0.02 ;>10 \mathrm{y}, p=0.03)$. This was found in ALL patients of both age groups.

Parameters of bone formation are shown in Table 5. Total osteoid volume, total osteoid surface, and osteoid seam thickness were not different, but again looking at active sites, there was a significant decrease in active osteoid surface $(<10 \mathrm{y}, p$ $=0.02 ;>10 \mathrm{y}, p=0.03)$ and in numbers of osteoblasts $(<10$ $\mathrm{y}, p=0.04 ;>10 \mathrm{y}, p=0.05)$ in ALL patients of both age groups.

Spearman correlation analysis gave a highly significant coefficient between the extent of the osteoblast-osteoid interface and the osteoid volume in all ALL patients and control subjects $<10 \mathrm{y}$. In control subjects $>10 \mathrm{y}$, no significant correlation was found. Putting together both age groups gave a high correlation in patients as well as in control subjects (Fig. 2).
The results are in the same range as those from some earlier histomorphometric studies (4-6), as well as from a recent comprehensive normative histomorphometric study in children (12), although the methods in this study differ slightly.

\section{DISCUSSION}

Two processes are controlling the growth dynamics of the skeleton of children. A continuous process of modeling results in a net gain of bone volume during growth. Throughout life, a cyclic process of remodeling maintains the bone integrity and mineral homeostasis (13). Growth hormones and somatomedins, thyroid hormone, and sex hormones are the most powerful systemic factors stimulating this modeling process, whereas parathyroid hormone (PTH), vitamin D metabolites, and calcitonin are involved in the mineral homeostasis. Trabecular bone and bone marrow are intermingled, and at this level, numerous local growth factors are involved in bone modeling and mineral homeostasis as well as hematopoiesis (14-16).

Control subjects were chosen because a bone marrow biopsy was included in their work-up. These children had various diagnoses, but demonstrated bone or bone marrow involvement made them ineligible. An adverse influence on bone turnover by means of systemic factors is not impossible, but in that case in general, bone turnover would be expected to be reduced and so the differences with the patient group would be even more significant. We preferred these control subjects to postmortem findings of victims of accidents or lethal, nonmalignant acute diseases because of the probability of rapid autolysis. The findings in our control subjects were in the same range as reported normative data of healthy children undergoing orthopedic surgery $(4-6,12)$.

From the results of this study, we conclude that ALL in children causes a reduced trabecular bone volume and reduced mean trabecular thickness. This may be due to a reduced number of osteoblasts leading to decreased osteoid formation, while osteoclastic resorption is going on at a reduced rate. The

Table 4. Histomorphometry results of ALL patients and control subjects: parameters of bone resorption

\begin{tabular}{lccc}
\hline & $\begin{array}{c}\text { Total erosion area } \\
(\% ; \text { median, range) }\end{array}$ & $\begin{array}{c}\text { Active erosion area } \\
(\% ; \text { median, range) }\end{array}$ & $\begin{array}{c}\text { No. of osteoclasts (per field } \\
\text { of vision; median, range) }\end{array}$ \\
\hline Patients $<10$ y $(n=18)$ & $22.4(11.4-47.8)$ & $3.6(0.1-11.2)$ & $2.7(0.0-6.4)$ \\
Controls $<10 \mathrm{y}(n=9)$ & $23.4(12.4-24.6)$ & $6.6(3.8-15.7)$ & $4.8(2.1-6.8)$ \\
$p$ value & NS & $<0.01$ & 0.02 \\
Patients $>10 \mathrm{y}(n=5)$ & $15.9(13.1-23.2)$ & $3.1(0.0-4.8)$ & $2.7(0.1-4.3)$ \\
Controls $>10 \mathrm{y}(n=6)$ & $23.4(11.6-27.6)$ & $8.7(1.2-15.0)$ & $6.4(1.2-8.3)$ \\
$p$ value & NS & 0.04 & 0.03 \\
\hline
\end{tabular}


Table 5. Histomorphometry results of ALL patients and control subjects: parameters of bone formation

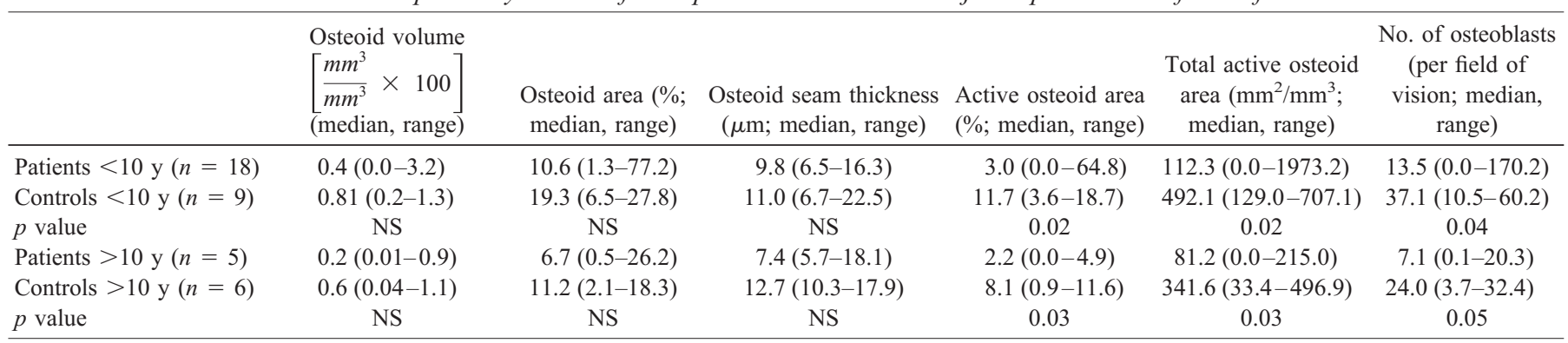

\section{A}

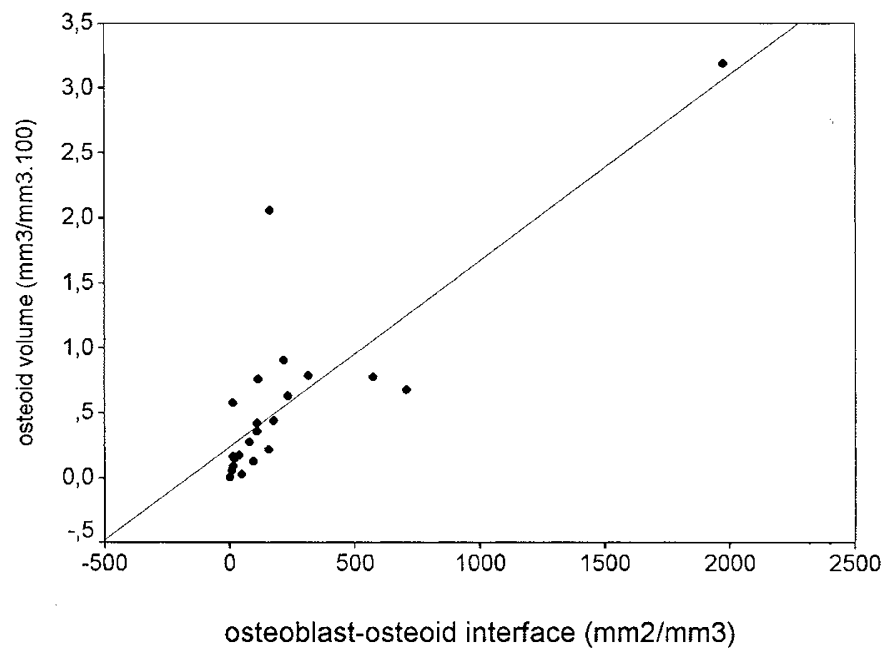

B

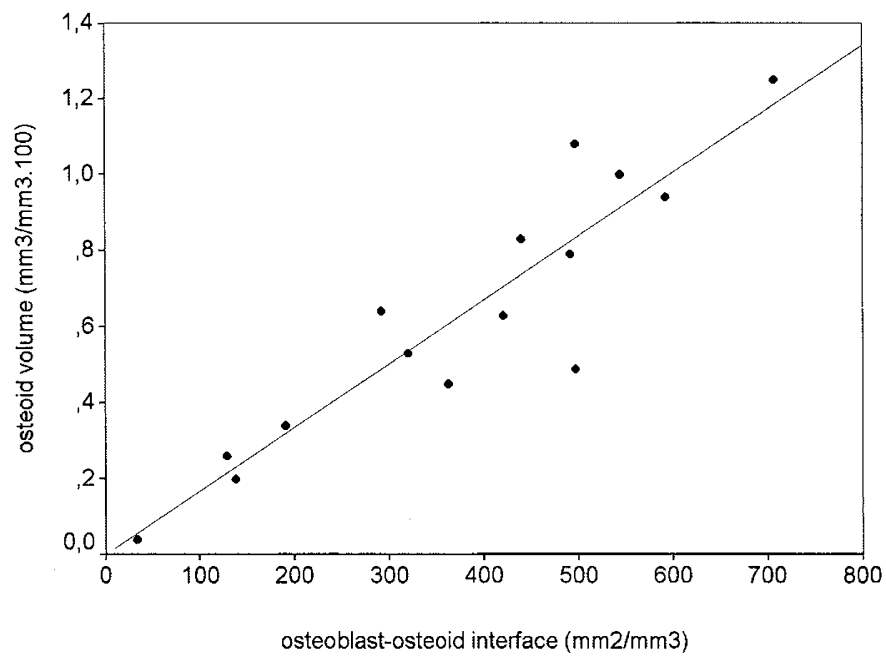

Figure 2. (A) Scatter plot of the extent of osteoblast-osteoid interface and osteoid volume in ALL patients of both age groups $(n=23)$. A strong correlation between these variables exists: Spearman correlation coefficient: $r_{s}$ $=0.97(p<0.01)$, arguing against the concept of osteomalacia. Regression analysis results in: $y=1.37 x-0.15$. $(B)$ Scatter plot of the extent of osteoblast-osteoid interface and osteoid volume in control subjects of both age groups $(n=15)$. Spearman correlation coefficient: $r_{\mathrm{s}}=0.91(p<0.01)$. Regression analysis: $\mathrm{y}=1.26 \mathrm{x}-0.04$.

modeling process as a whole is suppressed. Because trabeculae are reduced only in thickness and not in area, the skeletal impairment at diagnosis may be reversible.
A significantly reduced bone volume was found in ALL patients $<10$ y but not in ALL patients $>10$ y, possibly because of the small number of older children. We tried to solve this uncertainty by also considering results of bone densitometry. $Z$ scores for measurements of BMD (a measure comparable to morphometric bone volume) in both age groups were compared with normal age matched reference values and a comparison between the patients of the two age groups was made. The $z$ scores in ALL patients $<10$ y were significantly different $(p=0.03)$ from 0 (the mean of the reference population); the $z$ scores in ALL patients $>10$ y were not. Although again the number of older patients is small, the pattern is the same as in histomorphometry. The comparison between the two patient groups gave no difference. This supports the conclusion that in pubertal patients, the disturbance in bone remodeling is less serious, probably as a result of the strong stimulus on bone formation of sex hormones.

Active erosion was diminished in ALL patients of all ages, probably as part of the disturbed modeling process, in which the osteoblast has a central role in controlling the osteoclastic activity $(17,18)$. The remaining osteoclastic activity is apparently sufficient to maintain the mineral homeostasis. A reduced active osteoid area and number of osteoblasts was found in both age groups. These findings correspond with the reported changes in biochemical markers of bone turnover (3).

With respect to the detection of osteomalacia, histomorphometry has been reported to be more sensitive than the determination of biochemical markers and measurement of bone mineral content (19). In contrast to Halton et al. (2), we did not find indications of increased osteoid parameters or delayed mineralization (osteomalacia). A precise measurement of mineralization velocity can be obtained by tetracycline labeling, but the required time of $2 \mathrm{wk}$ makes it unsuitable for these patients. Nevertheless, even with static parameters, one can have an estimate of the actual bone formation.

Osteoblasts play a pivotal role in osteoid formation as well in subsequent mineralization. Delayed mineralization (osteomalacia) mostly is caused by insufficient availability of minerals (vitamin D deficiency, renal phosphate loss, etc.) and seldom by a direct inhibition (acidosis, fluoride overdose). PTH, 1,25-dihydroxy vitamin D, and calcitonin assays were done in 16 patients; PTH and calcitonin was performed in five other patients. All patients had serum calcium and phosphate measurements. Calcium and phosphate levels were in the vast majority within normal ranges (the lowest calcium level was associated with hypoproteinemia), as were $1,25(\mathrm{OH}) 2$-vitamin 
$\mathrm{D}_{3}$ and PTH (one unexplainable high outlier). This means an overall normal availability of minerals and as such an additional argument against osteomalacia. In normal bone throughout life, the most significant correlation exists between the extent of the osteoblast-osteoid interface and the osteoid volume, which is confirmed in our study (Fig. 2). Any mineralization delay disturbs this correlation (10). No correlation was found in controls $>10 \mathrm{y}$, which may be explained by the likely possibility that the numbers in these groups were too small to reach statistical significance.

Bone development is more age than sex dependent. When changes caused by puberty were taken into account, our patient numbers of this age group were too small to detect sex differences. Even in the large series of Glorieux et al. (12), significant differences between the sexes were found only in older children $(>14 \mathrm{y})$, mostly related to bone formation parameters.

In a series of 20 autopsies of patients who died of progressive ALL, also a generalized osteoporosis was found without increased osteoclastic activity and without osteomalacia. However, in these cases, leukemia treatment may have played a role as well (20). In our series no correlation was found with duration of complaints, ALL immunophenotype, or tumor burden (data not shown).

It is tempting to deduce from our data that ALL causes the bone formation impairment by changes in local growth factors on the bone marrow level. However, the same anomaly was found in a few control subjects as well, which leaves open the possibility of a systemic cause, such as, for example, the production of tumor necrosis factor- $\alpha(21,22)$, or disturbances of the bioavailability of insulin-like growth factors by production of insulin-like growth factor-binding proteins (23).

\section{REFERENCES}

1. Leheup B, Membre H, Gerard H, Olive D 1985 Lymphoblastic leukemia with osteopenia and vertebral compression fractures. J Pediatr 106:860

2. Halton JM, Atkinson SA, Fraher L, Webber CE, Cockshott WP, Tam C, Barr RD 1995 Mineral homeostasis and bone mass at diagnosis in children with acute lymphoblastic leukemia. J Pediatr 126:557-564

3. Sorva R, Kivivuori SM, Turpeinen M, Marttinen E, Risteli J, Risteli L, Sorva A 1997 Very low rate of type I collagen synthesis and degradation in newly diagnosed children with acute lymphoblastic leukemia. Bone 20:139-143
4. Schulz A, Delling G 1973 Histomorphometric preparation and technique. Determination of trabecular bone volume. In: Jaworski ZF, Klosevych S, Cameron E (eds) Proceedings of the First Workshop on Bone Morphometry. University of Ottawa Press, Ottawa, Ontario, Canada, pp 106-108

5. Schulz A, Delling G 1973 Age-related changes of bone resorption parameters in iliac crest trabecular bone. In: Jaworski ZF, Klosevych S, Cameron E (eds) Proceedings of the First Workshop on Bone Morphometry. University of Ottawa Press, Ottawa, Ontario, Canada, pp 161-162

6. Schulz A, Delling G 1973 Age-related changes of new bone formation. Determination of histomorphometric parameters of the iliac crest trabecular bone. In: Jaworski ZF, Klosevych S, Cameron E (eds) Proceedings of the First Workshop on Bone Morphometry. University of Ottawa Press, Ottawa, Ontario, Canada, pp 189-190

7. Gerrits PO, Eppinger B, Van Goor H, Horobin RW 1991 A versatile, low toxicity glycol methacrylate embedding medium for use in biological research, and for recovered biomaterials prostheses. Cells Materials 1:189-198

8. Page KM 1982 Bone and the preparation of bone sections. In: Bancroft JD, Stevens A (eds) Theory and Practice of Histological Techniques. Churchill Livingstone, Edinburgh, pp 323-324

9. Parfitt AM 1983 The stereologic basis of bone histomorphometry. Theory of quantitative microscopy and reconstruction of the third dimension. In: Recker RR (ed) Bone Histomorphometry: Techniques and Interpretations. CRC Press, Boca Raton, FL pp 53-87

10. Schenk RK 1979 Model cases from biological morphometry: bone tissue. In: Weibel ER (ed) Stereological Methods: Vol. 1, Practical Methods for Biological Morphometry. Academic Press, Harcourt Brace Jovanovich, London, pp 301-311

11. Parfitt AM, Drezner MK, Glorieux FH, Kanis JA, Malluche H, Menier PJ, Ott SM, Recker RR 1987 Bone histomorphometry: standardization of nomenclature, symbols and units. Report of the ASBMR Histomorphometry Nomenclature Committee. J Bone Miner Res 2:595-610

12. Glorieux FH, Travers R, Taylor A, Bowen JR, Rauch F, Norman M, Parfitt AM 2000 Normative data for iliac bone histomorphometry in growing children. Bone 26:103109

13. Jaworski ZF, Kimmel DB, Jee WSS 1983 Cell kinetics underlying skeletal growth and bone turnover. In: Recker RR (ed) Bone Histomorphometry: Techniques and Interpretation. CRC Press, Boca Raton, pp 226-227

14. Peck WA, Woods WL 1988 The cells of bone. In: Riggs BL, Melton III LJ (eds) Osteoporosis, Etiology, Diagnosis, and Management. Raven Press, New York, pp $11-19$

15. Vaughan J 1981 Osteogenesis and haematopoiesis. Lancet 2:133-136

16. Raisz LG 1981 What marrow does to bone. N Engl J Med 304:1485-1486

17. Horowitz MC, Coleman DL, Flood PM, Kupper TS, Jilka RL 1989 Parathyroid hormone and lipopolysaccharide induce murine osteoblast-like cells to secrete a cytokine indistinguishable from granulocyte-macrophage colony-stimulating factor. J Clin Invest 83:149-157

18. Löwik CWGM, Van der Pluijm G, Bloys H, Hoekman K, Bijvoet OLM, Aarden LA, Papapoulos SE 1989 Parathyroid hormone (PTH) and PTH-like protein (PLP) stimulate interleukin-6 production by osteogenic cells: a possible Role of Interleukin-6 in osteoclastogenesis. Bioch Biophys Res Commun 162:1546-1551

19. Arnala I 1991 Use of histological methods in studies of osteoporosis. Calcif Tissue Int 49:S31-S32

20. Thomas LB, Forkner CE, Frei E, Besse BE, Stabenau JR 1961 The skeletal lesions of acute leukemia. Cancer 14:608-621

21. Saarinen UM, Koskelo EK, Teppo AM, Siimes M 1990 Tumor necrosis factor in children with malignancies. Cancer Res 50:592-595

22. Kurzrock R, Kantarjian H, Wetzler M, Estrov E, Estey E, Troutman-Worden JU 1993 Ubiquitous expression of cytokines in diverse leukemias of lymphoid and myeloid lineage. Exp Hematol 21:80-85

23. Müller HL, Youngman OH, Lehrnbecher T, Blum WF, Rosenfeld RG 1994 Insulinlike growth factor-binding protein-2 concentrations in cerebrospinal fluid and serum of children with malignant solid tumors or acute leukemia. J Clin Endocrinol Metab 79:428-434 